\title{
Correction to: Qualitative and quantitative analysis of glenoid bone stock and glenoid version: inter-reader analysis and correlation with rotator cuff tendinopathy and atrophy in patients with shoulder osteoarthritis
}

\author{
Matthew J. Siebert ${ }^{1} \cdot$ Majid Chalian ${ }^{1} \cdot$ Arghavan Sharifi $^{1} \cdot$ Parham Pezeshk $^{1} \cdot$ Yin $\mathrm{Xi}^{1}$. \\ Parker Lawson ${ }^{1}$ - Avneesh Chhabra ${ }^{1,2,3,4,5}$
}

Published online: 21 February 2020

(C) ISS 2020

\begin{abstract}
Purpose Glenoid bone stock and morphology and rotator cuff muscle quality and tendon integrity affect the outcome of total shoulder arthroplasty. We hypothesized that glenoid bone loss correlates with rotator cuff muscle fatty infiltration (FI), tendinopathy, and atrophy.

Design Forty-three 3-D CT scans and MRIs of 43 patients (mean age 62 years; SD 13 years; range $22-77$ years) referred for primary shoulder pain were evaluated. Measurements of glenoid bone stock, version, and posterior humeral subluxation index (HSI) were assessed on an axial CT image reconstructed in the true scapular plane. Measurements utilized the Friedman line to approximate the pre-pathologic surface. Glenoid morphology was assigned by modified Walch classification. Rotator cuff FI, atrophy, and tendon integrity were assessed on corresponding MRIs.

Results There was a very strong negative correlation between increasing glenoid version and HSI $(r=-0.908$; $p<0.0001)$. There was a moderately negative correlation between anterior bone loss and HSI $(r=-0.562 ; p<0.0001)$ and a moderately positive correlation between posterior bone loss and HSI $(r=0.555 ; p<0.0001)$. Subscapularis muscle FI correlated moderately with increased anterior and central bone loss and increased humeral head medialization $(r=$ $0.512, p=0.0294 ; r=0.479, p=0.033 ; r=0.494, p=0.0294$, respectively). Inter-observer reliability (intra-class correlation coefficient [ICC] and kappa) was good to excellent for all measurements and grading.

Conclusion Glenoid anteversion and anterior and posterior bone loss are associated with varying HSI. Subscapularis muscle FI, not tendon integrity, correlates to anterior and central glenoid erosion. The study adds evidence that neither rotator cuff tendinopathy nor muscle atrophy exhibits a significant relationship to HSI.
\end{abstract}

Keywords Glenoid · Bone stock · Rotator cuff · Atrophy $\cdot$ Subluxation

The original version of this article which can be found at https:// doi.org/10.1007/s00256-020-03377-0 is not the final version of the manuscript. To avoid any misinterpretation, the whole article is published below in full with the correct data.

The online version of the original article can be found at https://doi.org/10. 1007/s00256-020-03377-0

Avneesh Chhabra

avneesh.chhabra@utsouthwestern.edu

Radiology, UT Southwestern Medical Center, Dallas, TX, USA

2 Orthopedics, University of Texas Southwestern Medical Center, 5323 Harry Hines Blvd, Dallas, TX 75390, USA
3 Radiology and Orthopedic Surgery, UT Southwestern Medical Center, Dallas, TX, USA

4 Johns Hopkins University, Baltimore, MD, USA

5 Walton Center of Neurosciences, Liverpool, UK 


\section{Introduction}

Shoulder osteoarthritis (OA) is a significant source of morbidity, present in up to $32.8 \%$ of patients over the age of 60 years [1,2]. In 2013, the total medical expenditures and earning losses attributable to OA (of all joints) were estimated at $\$ 303.5$ billion in the USA [3]. Contributing to these medical expenditures is the number of patients undergoing arthroscopic resurfacing, total anatomic (or traditional) shoulder arthroplasty (TSA), reverse shoulder arthroplasty (RSA), or hemiarthroplasty (HA). In 2011, about 66,485 patients underwent shoulder arthroplasty with 21,692 cases of RSA; 29,359 TSA; 15,434 HA and the utilization of these interventions has continued to increase yearly $[4,5]$.

Studies have shown association of factors, such as glenoid retroversion, and rotator cuff muscle atrophy with poor clinical outcomes including premature hardware loosening and recurrent subluxation of the prosthetic humeral head following anatomic TSA [6,7]. The relationship between both soft tissue and preoperative bone pathologies was recently assessed by Donohue et al. who found an association between high-grade rotator cuff muscle fatty infiltration and more severe modified Walch classification subtype, increased joint-line medialization, and increased glenoid retroversion [8]. However, the full spectrum of Walch classification was not studied and inter-rater performance was not assessed.

In the light of above literature, considerable gaps in knowledge still exist. The relationships between glenoid bone stock, bone loss, and pre-surgical humeral head subluxation index (HSI) and rotator cuff tendon tears or rotator cuff muscle fatty infiltration and atrophy has not been defined. Different variants of glenoid morphology, as illustrated by the commonly used Walch classification, and its association with rotator cuff pathology need further assessment.

The goal of the present study is to assess the relationship of dynamic soft tissue and static bony stabilizers of the glenohumeral joint in the setting of $\mathrm{OA}$ using 3-dimensional CT (3DCT) and MRI prior to the joint replacement. We hypothesized that the severity of glenoid bone loss correlates with full thickness or higher grade rotator cuff tendon tears on imaging and with worsening severity of fatty infiltration and atrophy of the rotator cuff musculature.

\section{Methods}

This was a cross-sectional study performed in compliance with HIPAA regulations and was approved by our tertiary care institutional review board. Informed consent was waived due to the retrospective nature of the study. The 3DCT scans of 418 shoulders in 398 patients who presented with a primary complaint of shoulder pain were reviewed. Only shoulders with primary glenohumeral OA and concurrent $\mathrm{CT}$ and magnetic resonance imaging (MRI) were included in the study. Patients with MRI obtained greater than 3 months after CT were excluded from the study. Shoulders with evidence of current or recent trauma (either fracture or soft tissue injury), evidence of history of prior surgery for rotator cuff repair or arthroplasty, inflammatory arthropathy, or osteonecrosis were excluded from the study. Of the 418 shoulders reviewed, 43 shoulders of 43 patients met inclusion criteria (Fig. 1). There were 21 male and 22 female patients with a mean age of 62 years old (range 22-77 years of age; SD 13 years).

\section{Imaging and slice reconstructions}

All CT scans were obtained at the patient's index presentation with the arm at the patient's side, palm facing up, and the shoulder in external rotation with beam collimation of $0.6 \mathrm{~mm}$ (isotropic resolution) to facilitate $3 \mathrm{D}$ reconstruction and proper assessment of humeral head subluxation. The scans were obtained on multislice (64-256 slice) scanners using automated iterative reconstruction with low radiation dose profile $(\mathrm{KVp}=100-120$ and $\mathrm{mAs}=250-300)$. All scans included the entirety of the scapula. The scans were uploaded into Aquarius iNtuition, a CT image 3D reconstruction software (TeraRecon, v.4.4.13.P2.; Foster City, CA). The shoulder MRI scans were obtained on 1.5T (26 patients) and 3T (17 patients) scanners. Standard protocols with multiplanar intermediate weighted scans $(\mathrm{TR}=3500-4500 \mathrm{~ms}, \mathrm{TE}=35$ $45 \mathrm{~ms}$, slice thickness $3.5-4.0 \mathrm{~mm}$, frequency elective fat suppression) with and without fat suppression in all three orthogonal orientations were obtained using a multi-channel shoulder coil in the supine position.

Images without fat suppression were used for assessment of fatty infiltration.

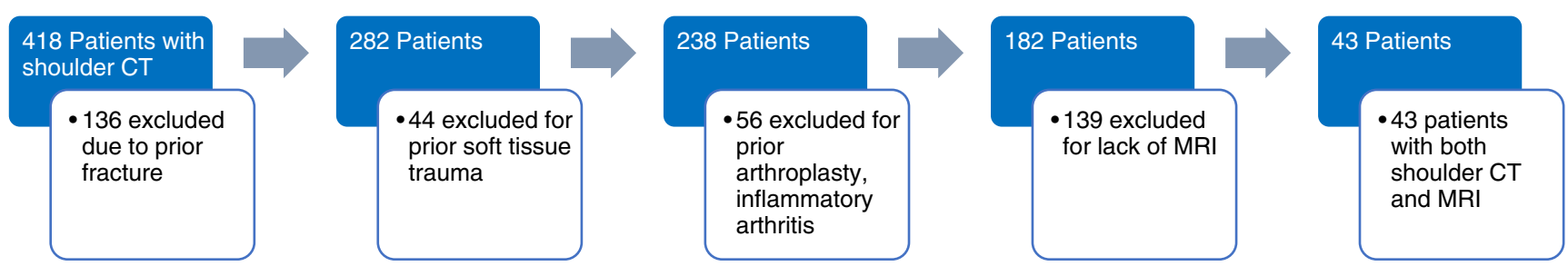

Fig. 1 Of the 418 patients identified with shoulder CT, 43 patients met inclusion criteria and were included in the study 


\section{Bony measurements}

Prior to the acquisition of measurements, scans were reconstructed in the scapular plane, defined by the inferior angle, scapular trigonum, and glenoid midpoint as previously described [8]. All CT measurements were made on an axial slice perpendicular to the scapular plane at the level of the junction of the base of the coracoid and spine of the scapula. Two trained 4th year medical student readers under the supervision of an experienced, board-certified, musculoskeletal fellowshiptrained radiologist obtained the measurements for inter-reader analysis. Initial training included 10 cases with different measurements. After training, these cases were repeated and included in the final data set. Random checks were later performed by the same radiologist for consistency of the measurements. Intrareader measurements on all the 43 cases were again repeated by one of the students blinded to the initial measurements, 3 weeks after the initial measurements were completed.
Each case was classified according to glenoid morphology in accordance with the criteria as detailed in the modified Walch classification system [9]. The assignment to each class was performed by both independent reads and consensus reading by two experienced, board-certified, fellowshiptrained musculoskeletal radiologists.

Glenoid version was measured by the Friedman line method, as this method was found to have greater inter-reader reliability than the popular scapular body method [10]. A line perpendicular to the Friedman line, the line connecting the medial border of the scapular body and midpoint of the glenoid fossa, was first placed (Fig. 2a) [10]. Version was measured as any angular deviation in the line connecting the anterior and posterior endpoints of the glenoid fossa from the Friedman line, with anterior wear representing anteversion, and posterior wear representing retroversion. Bone loss was measured by placing a line perpendicular to the Friedman line at the most lateral edge of the glenoid fossa, thus
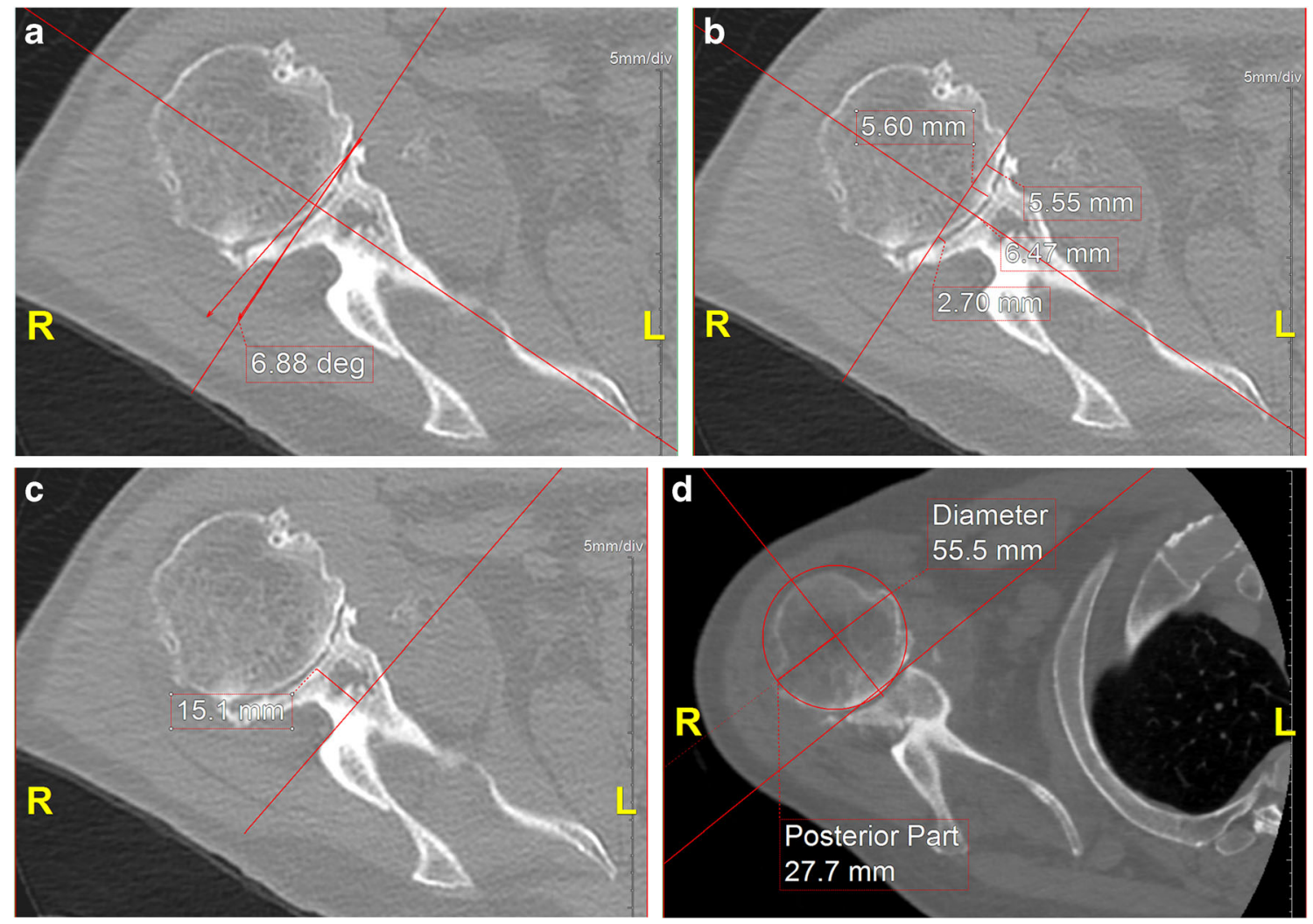

Fig. 2 Glenoid measurement techniques. a Glenoid version by Friedman line method. The Friedman line is placed perpendicular to a line connecting the medial border of the scapular body and midpoint of the glenoid fossa. Version is measured as any angular difference from the Friedman line with anterior wear representing anteversion and posterior wear representing retroversion. This figure shows $6.88^{\circ}$ of glenoid anteversion. b Glenoid bone loss and humeral head medialization by paleoglenoid line method. The paleoglenoid line was approximated by placing a line at the most lateral lip of the glenoid, perpendicular to Friedman line. Bone loss was measured at $5 \mathrm{~mm}$ from the anterior and posterior rims of the glenoid fossa, and at the midpoint of the glenoid

fossa. Humeral head medialization was measured at the apex of the humeral head medial to the paleoglenoid line. In this figure, anterior bone loss $=5.55 \mathrm{~mm}$; central bone loss $=6.47 \mathrm{~mm}$; posterior bone loss $=2.70 \mathrm{~mm}$; and humeral head medialization $=5.60 \mathrm{~mm}$. $\mathbf{c}$ Depth of glenoid vault. The depth of the glenoid vault was measured as the depth from the surface of the glenoid fossa at the glenoid midpoint to a line at the junction of the spinous process and glenoid. The depth of the glenoid vault displayed here is $15.1 \mathrm{~mm}$. d Humeral subluxation index (HSI) by the Walch method with modification. The HSI of the displayed patient is $27.7 \mathrm{~mm} / 55.5 \mathrm{~mm}=0.49$ 
approximating the native, or paleoglenoid, surface. When osteophytes were present, the axial slices above and below the junction of the base of the coracoid and spine of the scapula were analyzed to determine the true paleoglenoid surface; these cases were confirmed by the experienced, board-certified, fellowship-trained musculoskeletal radiologist above. Measures of bone loss were taken at $5 \mathrm{~mm}$ from the anterior and posterior edges of the glenoid rim, and the glenoid center point (Fig. 2b). Humeral head medialization was measured at the apex of medialization relative to the paleoglenoid line (Fig. 2b). The depth of the glenoid vault was measured as the depth from the surface of the glenoid fossa at the glenoid midpoint to a line at the junction of the spinous process and glenoid (Fig. 2c).

HSI was measured as described by Walch et al. with minor modification [11]. Rather than measuring the diameter of the humeral head at the medial $1 / 3$ of the humeral head, we measured the diameter of the circle of best fit corresponding to the humeral head articular surface to reduce variability due to the level or subjectivity in determining the medial $1 / 3$ point. Because inter-reader consensus has been less than initially reported using this method in numerous studies, we ensured proper positioning of region of interest measurements in all shoulders as above $[12,13]$. Using Mose rings, a curve of best fit was applied to the articular surface of the humeral head at the level where our other were measurements taken. HSI was then calculated using the Mose ring of best fit in the fashion proposed by Walch et al. [11]. (Fig. 2).

\section{Rotator cuff tear and muscle grading}

All rotator cuff scoring was performed independently by the above two board-certified, musculoskeletal fellowship-trained radiologists on a PACS (picture archiving and communication system, iSITE, Philips, Best, Netherlands) workstation. Rotator cuff tendinopathy and tears were graded on oblique coronal and sagittal fat-saturated proton density (PD) MR images as follows: the normal tendons received a score of 0 ; tendinosis or low-grade ( $<50 \%$ ) partial-thickness tears, 1 ; high grade $(>50 \%)$ partialthickness or full-thickness partial width tears, 2; and full-thickness, full-width tears, 3 . Rotator cuff muscle fatty infiltration was graded according to criteria detailed in the Goutallier classification [14]. Rotator cuff muscle atrophy was evaluated on oblique sagittal non-fat-saturated PD MR images according to the classification of Warner et al. [15]. Medial to the level of coracoid process, where a Y view is formed, a line is drawn from the edge of the coracoid to the inferior scapular tip, from the inferior tip of the scapula to the scapular spine at the junction of infraspinatus muscle and posterior belly of the deltoid muscle, and from the tip of the scapular spine to the coracoid process (Fig. 3). The grading system for atrophy was as follows: no atrophy = muscle completely fills its fossa, and the outer contour is convex; mild atrophy $=$ muscle's outer contour is flat compared with its fossa and is even to the line; moderate atrophy = muscle's outer contour is concave into the fossa below the line; and severe atrophy $=$ muscle is barely apparent in its fossa.

\section{Statistical analysis}

Weighted Kappa, simple Kappa, and intra-class correlations were used to assess the inter-reader agreements when appropriate. Inter-reader agreement was classified as excellent $(>0.80)$, good $(0.61-0.80)$, moderate $(0.41-$ $0.60)$, fair $(0.20-0.40)$, and poor $(<0.20)$ using $95 \% \mathrm{CI}$ (confidence intervals). All measurements were averaged across all reads. Kruskal-Wallis tests were used to test the difference in the CT measurements among different Walch categories. Pearson correlation coefficient between the CT measurements and MR measurement was calculated. Bonferroni correction was used for multiple comparisons. All analyses were done in SAS 9.4 (SAS Institute Inc., Cary, NC).

\section{Results}

\section{Types of glenoids and intra-reader and inter-reader reliability}

Of the 43 shoulders reviewed, there were $9 \mathrm{~A} 1,13 \mathrm{~A} 2,1 \mathrm{~B} 1,7$ B2, 3 B3, and 10 D Walch type glenoids. The intra-observer reliability of all measurements was near perfect with all ICC greater than or equal to 0.97 (lower limit of $95 \%$ CI greater than 0.938) (Table 1).

The inter-reader reliability of glenoid version was excellent with ICC of 0.88 (95\% CI, 0.79-0.93). Anterior bone loss, central bone loss, and humeral head medialization showed good reliability with ICCs of $0.78,0.69$, and 0.78 , respectively (95\% CI $0.61-0.87 ; 0.50-0.82 ; 0.64-0.87$ ). Posterior bone loss showed good inter-reader reliability, ICC 0.76 (95\% CI, 0.59-0.86), and HSI was excellent with ICC of 0.94 (95\% CI, 0.90-0.97) (Table 2). The inter-reader reliability of Walch classification, rotator cuff muscle fatty infiltration grading, and rotator cuff muscle atrophy were all good to excellent. The reliability of assessing the severity of rotator cuff tendon tears was good with ICCs ranging 0.60-0.71. (Table 2).

\section{Glenoid morphology, measurements, and bony correlations}

Glenoid version differed significantly $(p=0.00077)$ between the modified Walch classes with significantly greater anteversion in D type glenoids (median, 11.63 ${ }^{\circ}$ anteversion; standard deviation, $10.01^{\circ}$ ) than $\mathrm{A} 1, \mathrm{~A} 2$, and B2 glenoids. Similarly, D type glenoids had 
Fig. 3 Measurement of rotator cuff muscle atrophy. a

Representing no atrophy in any muscles. The contours of all rotator cuff muscles are convex and all fossa are filled completely. b Mild atrophy in the supraspinatus muscle with the contour of the supraspinatus muscle flat with its fossa. $\mathbf{c}$ Moderate atrophy in supraspinatus muscle and severe atrophy in infraspinatus muscle. The supraspinatus muscle is concave within its fossa and the infraspinatus muscle is barely apparent. $\mathbf{d}$ Moderate atrophy in supraspinatus and infraspinatus muscles. The supraspinatus and infraspinatus muscles are both concave within their respective fossa
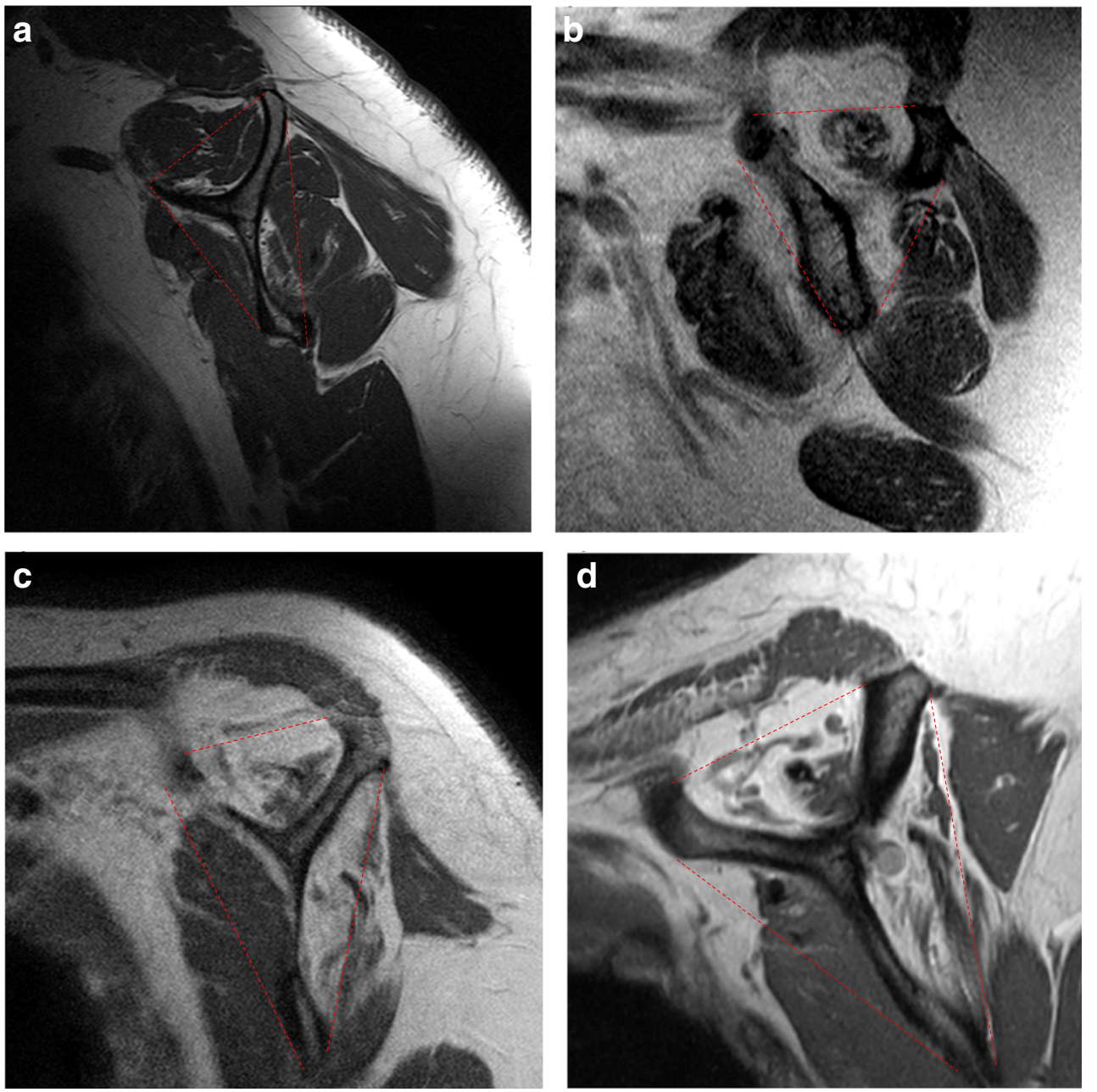

significantly greater anterior bone loss (median, $5.99 \mathrm{~mm}$; standard deviation, $2.09 \mathrm{~mm}$ ) compared with all other Walch classes $(p=0.0148)$. Type D glenoids also showed significantly decreased HSI compared with other modified Walch classes $(p=0.011$; median, 0.44; standard deviation 0.12) (Table 3). Type B3 glenoids exhibited significantly greater retroversion, central and

Table 1 Intra-reader reliability scores for all bony glenoid measurements

\begin{tabular}{llll}
\hline Measurement & ICC & \multicolumn{2}{l}{$95 \%$ CI } \\
\hline Glenoid version & 0.99 & 0.99 & 0.99 \\
Anterior bone loss & 0.99 & 0.98 & 0.99 \\
Central bone loss & 0.99 & 0.99 & 0.99 \\
Posterior bone loss & 0.99 & 0.99 & 0.99 \\
Humeral head medialization & 0.99 & 0.99 & 0.99 \\
Depth of glenoid vault & 0.97 & 0.93 & 0.98 \\
Humeral head subluxation index & 0.99 & 0.98 & 0.99 \\
\hline
\end{tabular}

posterior bone loss, humeral head medialization, and HSI compared with other classes, but were excluded from analysis due to low number found in the patient sample $(n=3)$.

There was a strong positive correlation between glenoid anteversion and anterior bone loss $(r=0.645 ; p<0.0001)$ and a moderate negative correlation between glenoid retroversion and posterior bone loss $(r=-0.613 ; p<0.0001)$. There was a moderate negative correlation between anterior bone loss and HSI $(r=-0.562 ; p<0.0001)$ and a moderate positive correlation between posterior bone loss and HSI $(r=0.555$; $p<0.0001)$. There was a very strong negative correlation between increasing glenoid anteversion and HSI $(r=-0.908$; $p<0.0001)$.

\section{Glenoid shapes, measurements, and correlations with rotator cuff pathology}

No difference was found in rotator cuff muscle fatty infiltration and atrophy scores or tendon tear severity between the modified Walch types (Tables 4 and 5). However, increased 
Table 2 Inter-reader reliability scores for all bony measurements, Modified Walch classification, rotator cuff muscle fatty infiltration and atrophy and rotator cuff tendon tear severity

\begin{tabular}{llll}
\hline Measurement & ICC & $95 \%$ CI & \\
\hline Glenoid version & 0.88 & 0.79 & 0.93 \\
Anterior bone loss & 0.78 & 0.61 & 0.87 \\
Central bone loss & 0.69 & 0.50 & 0.82 \\
Posterior bone loss & 0.76 & 0.59 & 0.86 \\
Humeral head medialization & 0.78 & 0.64 & 0.87 \\
Humeral subluxation index & 0.94 & 0.90 & 0.97 \\
Depth of glenoid vault & 0.58 & 0.35 & 0.75 \\
Fatty infiltration of infraspinatus & 0.85 & 0.73 & 0.96 \\
Fatty infiltration of subscapularis & 0.75 & 0.61 & 0.90 \\
Fatty infiltration of supraspinatus & 0.85 & 0.74 & 0.96 \\
Fatty infiltration of teres minor & 0.83 & 0.70 & 0.95 \\
Muscle atrophy infraspinatus & 0.79 & 0.67 & 0.91 \\
Muscle atrophy subscapularis & 0.83 & 0.66 & 0.99 \\
Muscle atrophy supraspinatus & 0.77 & 0.64 & 0.91 \\
Muscle atrophy teres minor & 0.80 & 0.64 & 0.97 \\
Tendon tear severity infraspinatus & 0.71 & 0.50 & 0.92 \\
Tendon tear severity subscapularis & 0.63 & 0.37 & 0.88 \\
Tendon tear severity supraspinatus & 0.69 & 0.52 & 0.87 \\
Tendon tear severity teres minor & 0.60 & 0.36 & 0.85 \\
Walch classification & 0.82 & 0.69 & 0.95 \\
\hline
\end{tabular}

Goutallier's score in the subscapularis muscle correlated moderately with increased anterior and central bone loss as well as increased humeral head medialization $(r=0.512, p=0.0294$; $r=0.479, p=0.033 ; r=0.494, p=0.0294$, respectively) (Tables 4 and 5).

\section{Discussion}

The present study sought to evaluate the relationships between pathologic changes in the glenoid bone stock and rotator cuff muscle and tendon pathology, both qualitatively and quantitatively. It should be noted the study sample included 10 Walch D glenoids, a relatively large number of this less common morphology. The Walch D is defined by Bercik et al. as being anteverted $\left(\geq 5^{\circ}\right)$ with anterior humeral head subluxation $[9$, 16]. Based on these criteria, we found 10 of the glenoids studied to be Walch $\mathrm{D}$. The relatively high incidence of this morphology may be due to the patient population studied, all of whom were treated by a well-known shoulder surgeon whose practice may attract more advanced shoulder pathology. The inclusion of these 10 Walch D glenoids yields significant findings not reported in previous literature which did not report on Walch D glenoids [8]. Our study cohort did however not have sufficient numbers of B1, B2, or B3 glenoids to corroborate or add to published findings regarding these morphologies [8].

Intra-observer reliability of the proposed glenoid bone loss protocol was near perfect, likely owing to its similarities to the Friedman line method of measuring glenoid version, which has been found to have similarly exceptional reliability [10]. Inter-observer reliability was good to excellent for all measurements performed, again, similar to published results [10]. The reformatting of images to the scapular plane and measuring on axial slice perpendicular to that plane likely contributed to the high reliability of this method, as did the use of a standardized level in the axial plane for measurement [17]. This validates 3DCT as an excellent imaging method for evaluation of such parameters.

The relationship between the glenoid version and HSI remains controversial. Despite prior studies [18, 19] suggesting that there is no relationship between glenoid version and humeral head subluxation in patients with posterior shoulder instability, we found increasing anteversion related to decreasing HSI $(p<0.0001)$. This finding is supported by other studies demonstrating the relationship between glenoid anteversion and frank anterior dislocation of the shoulder $[20,21]$. In addition, we found anterior bone loss correlating to decreased HSI $(p<0.0001)$. Though we did not find a direct correlation between glenoid retroversion and increasing HSI,

Table 3 Mean values of all glenoid measurements for each Walch class

\begin{tabular}{|c|c|c|c|c|c|c|c|c|c|c|c|c|}
\hline & \multicolumn{2}{|l|}{ A1 } & \multicolumn{2}{|l|}{ A2 } & \multicolumn{2}{|l|}{ B1 } & \multicolumn{2}{|l|}{ B2 } & \multicolumn{2}{|l|}{ B3 } & \multicolumn{2}{|l|}{$\mathrm{D}$} \\
\hline & Median & IQR & Median & IQR & Median & IQR & Median & IQR & Median & IQR & Median & IQR \\
\hline Glenoid version in degrees & -2.69 & 4.34 & -4.28 & 12.09 & -8.15 & 0 & -4.1 & 17.72 & -20.13 & 7.77 & 11.63 & 8.56 \\
\hline Anterior bone loss (mm) & 2.11 & 1.2 & 2.54 & 2.87 & 2.08 & 0 & 1.89 & 1.5 & 2.77 & 1.67 & 5.99 & 2.85 \\
\hline Central bone loss (mm) & 2.95 & 2.14 & 3.52 & 4.07 & 3.75 & 0 & 3.16 & 3.17 & 5.7 & 2.57 & 4.34 & 2.45 \\
\hline Posterior bone loss (mm) & 1.57 & 0.65 & 2.85 & 3.36 & 4.02 & 0 & 4.14 & 5.87 & 9.66 & 4.89 & 1.81 & 2.47 \\
\hline Humeral head medialization (mm) & 0 & 0.23 & 1.95 & 3.39 & 2.39 & 0 & 1.87 & 6.02 & 5.69 & 4.49 & 3.29 & 4.17 \\
\hline Depth of glenoid bone stock in $\mathrm{mm}$ & 17.47 & 1.9 & 19.6 & 3.9 & 18.27 & 0 & 18.6 & 3.63 & 18.77 & 5.84 & 18.23 & 5.5 \\
\hline Humeral subluxation index & 0.55 & 0.08 & 0.56 & 0.1 & 0.63 & 0 & 0.63 & 0.23 & 0.74 & 0.11 & 0.44 & 0.15 \\
\hline
\end{tabular}


Table 4 Correlation between glenoid morphology, glenoid measurements, and rotator cuff pathology

\begin{tabular}{|c|c|c|c|c|c|c|c|c|}
\hline Fatty Infiltration of Infraspinatus - & 0.1946 & 0.175 & 0.1654 & 0.1395 & -0.088 & 0.0153 & 0.075 & \multirow{3}{*}{-0.4} \\
\hline Fatty Infiltration of Subscapularis - & 0.5112 & 0.4781 & 0.3842 & 0.4933 & 0.0934 & 0.0914 & -0.106 & \\
\hline Fatty Infiltration of Supraspinatus - & 0.2701 & 0.1538 & 0.0196 & 0.0388 & -0.204 & 0.1252 & -0.194 & \\
\hline Fatty Infiltration of Teres Minor - & 0.2062 & 0.126 & 0.2104 & 0.1204 & -0.077 & 0.0726 & 0.0168 & \multirow{6}{*}{ 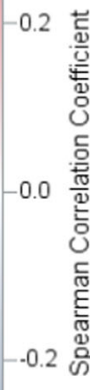 } \\
\hline Muscle Atrophy Infraspinatus - & 0.1571 & 0.094 & 0.0708 & 0.0425 & -0.236 & -0.018 & -0.053 & \\
\hline Muscle Atrophy Subscapularis & 0.1932 & 0.1701 & 0.0088 & 0.153 & -0.192 & 0.1001 & -0.141 & \\
\hline Muscle Atrophy Supraspinatus - & 0.1414 & -0.013 & -0.077 & -0.082 & -0.249 & 0.154 & -0.118 & \\
\hline Muscle Atrophy Teres Minor - & 0.3213 & 0.2999 & 0.2931 & 0.3146 & -0.249 & -0.018 & 0.0316 & \\
\hline Tendon Tear Severity Infraspinatus & 0.1989 & 0.1613 & 0.0767 & 0.0269 & -0.221 & 0.1225 & -0.026 & \\
\hline Tendon Tear Severity Subscapularis & 0.2205 & 0.1974 & 0.1178 & 0.1574 & 0.0283 & 0.1306 & -0.128 & \\
\hline Tendon Tear Severity Supraspinatus - & -0.091 & -0.131 & -0.066 & -0.127 & -0.118 & 0.038 & -0.045 & -0.4 \\
\hline \multirow[t]{2}{*}{ Tendon Tear Severity Teres Minor - } & -0.092 & -0.087 & -0.099 & 0.0325 & -0.515 & 0.0612 & -0.096 & \\
\hline & $\begin{array}{c}\text { Anterior } \\
\text { Glenoid } \\
\text { Bone Loss }\end{array}$ & $\begin{array}{c}\text { Central } \\
\text { Glenoid } \\
\text { Bone Loss }\end{array}$ & $\begin{array}{c}\text { Posterior } \\
\text { Glenoid } \\
\text { Bone Loss }\end{array}$ & $\begin{array}{c}\text { Humeral } \\
\text { Head } \\
\text { Subluxation }\end{array}$ & $\begin{array}{c}\text { Glenoid } \\
\text { Bone Stock }\end{array}$ & $\begin{array}{l}\text { Glenoid } \\
\text { Version }\end{array}$ & $\begin{array}{l}\text { Humeral } \\
\text { Head } \\
\text { Subluxation } \\
\text { Index }\end{array}$ & \\
\hline
\end{tabular}

Fatty infiltration of the subscapularis muscle correlated moderately with humeral head medialization and anterior and central glenoid bone loss. More severe tears of the teres minor tendon were moderately associated with decreased glenoid bone stock

there was a correlation between glenoid retroversion and posterior bone loss $(p<0.0001)$ and a second relationship between posterior bone loss and HSI $(p<0.0001)$. Thus, glenoid retroversion appears to be indirectly related to increasing HSI.
We found that the HSI did not correlate to worsening rotator cuff muscle fatty infiltration, atrophy, or tendinopathy ( $p>0.80$ in all comparisons). Only glenoid anteversion, anterior bone loss, and posterior bone loss exhibited any direct

Table 5 Significance of correlations between glenoid morphology, glenoid measurements, and rotator cuff pathology

\begin{tabular}{|c|c|c|c|c|c|c|c|}
\hline $\begin{array}{l}p \text { value after } \\
\text { FDR correction }\end{array}$ & $\begin{array}{l}\text { Anterior } \\
\text { glenoid } \\
\text { bone loss }\end{array}$ & $\begin{array}{l}\text { Central } \\
\text { glenoid } \\
\text { bone loss }\end{array}$ & $\begin{array}{l}\text { Posterior } \\
\text { glenoid } \\
\text { bone loss }\end{array}$ & $\begin{array}{l}\text { Humeral head } \\
\text { medialization }\end{array}$ & $\begin{array}{l}\text { Depth of glenoid } \\
\text { bone stock }\end{array}$ & $\begin{array}{l}\text { Glenoid } \\
\text { version }\end{array}$ & $\begin{array}{l}\text { Humeral head } \\
\text { subluxation } \\
\text { index }\end{array}$ \\
\hline Fatty infiltration of infraspinatus & 0.8045 & 0.8385 & 0.8385 & 0.8385 & 0.8532 & 0.9468 & 0.8532 \\
\hline Fatty infiltration of subscapularis & 0.0294 & 0.033 & 0.2209 & 0.0294 & 0.8532 & 0.8532 & 0.8532 \\
\hline Fatty infiltration of supraspinatus & 0.7362 & 0.8385 & 0.9468 & 0.9468 & 0.8045 & 0.8385 & 0.8045 \\
\hline Fatty infiltration of teres minor & 0.8045 & 0.8385 & 0.8045 & 0.8385 & 0.8532 & 0.8532 & 0.9468 \\
\hline Muscle atrophy infraspinatus & 0.8385 & 0.8532 & 0.8532 & 0.9468 & 0.8045 & 0.9468 & 0.9156 \\
\hline Muscle atrophy subscapularis & 0.8045 & 0.8385 & 0.9566 & 0.8385 & 0.8045 & 0.8532 & 0.8385 \\
\hline Muscle atrophy supraspinatus & 0.8385 & 0.9469 & 0.8532 & 0.8532 & 0.8045 & 0.8385 & 0.8385 \\
\hline Muscle atrophy teres minor & 0.5419 & 0.587 & 0.587 & 0.5419 & 0.8045 & 0.9468 & 0.9468 \\
\hline Tendon tear severity infraspinatus & 0.8045 & 0.8385 & 0.8532 & 0.9468 & 0.8045 & 0.8385 & 0.9468 \\
\hline Tendon tear severity subscapularis & 0.8045 & 0.8045 & 0.8385 & 0.8385 & 0.9468 & 0.8385 & 0.8385 \\
\hline Tendon tear severity supraspinatus & 0.8532 & 0.8385 & 0.8731 & 0.8385 & 0.8385 & 0.9468 & 0.9468 \\
\hline Tendon tear severity teres minor & 0.8532 & 0.8532 & 0.8532 & 0.9468 & 0.0294 & 0.8916 & 0.8532 \\
\hline
\end{tabular}

The correlations between fatty infiltration of the subscapularis muscle and humeral head medialization, anterior and central glenoid bone loss were significant. There was also a significant relationship between severity of teres minor tendon tears and depth of glenoid bone stock. All other relationships were not statistically significant 
relationships to HSI. It would appear then that actual glenoid bone loss or malorientation is more responsible for anterior and posterior humeral head subluxation rather than rotator cuff pathology. This is keeping with the physiology of the glenohumeral joint. The primary mechanism by which the humeral head is centered within the glenoid cavity is the principle of concavity-compression [22]. As such, any anterior or posterior bone loss alters the concavity of the glenoid cavity and thus, static destabilization, especially those decreasing the depth and curvature, increases the likelihood of decentering of the humeral head. Indeed, multiple reports have been made of the impact of anterior bone loss and frank anterior shoulder dislocation [20, 21]. Our findings support these prior findings in adding additional evidence that it is glenoid bone loss that correlates most directly with pathologic humeral head subluxation, both anteriorly and posteriorly. However, it should be noted that our results are applicable to middle aged and older population deemed to undergo shoulder replacement. We did not study the spectrum of rotator cuff pathology as it pertains to a younger population.

We also found significant relationships between fatty infiltration of the subscapularis muscle and anterior and central glenoid bone loss. The cross-sectional design of our study is insufficient to determine the temporal sequence of muscle atrophy versus bone loss. However, infants born with obstetric brachial plexus lesions commonly exhibit internal rotation contracture, posterior glenoid bone loss, and posterior subluxation as a result of imbalanced forces secondary to posterior rotator cuff weakness or paralysis [23, 24]. Our results, with increased anterior and central bone loss correlating to fatty infiltration, and hence weakness, of the subscapularis muscle may suggest a similar but opposite imbalance of forces. The relationship between anterior rotator cuff muscle weakness and anterior and central bone loss suggests a possible utility in targeted pre and post-operative strengthening of the subscapularis. However, additional studies are required to determine if this muscular atrophy is an independent factor affecting the outcome of TSA.

It is possible that our inclusion criteria, not limited to patients with posterior instability, resulted in a patient population with different glenoid characteristics than previously studied. Indeed, prior studies typically only included patients with pathologic retroversion, not anteversion. Most likely, however, the method used in our study to measure HSI was less affected by the level at which it was measured than the technique used in prior studies. The use of a circle of best fit, approximated to the articular surface of the humeral head, yields a circle of identical radius regardless of the level it is measured, except for any manual error. Thus, this technique allowed for measurement of subluxation unaffected by level, which likely explains our observation of relationship between glenoid version and humeral subluxation where prior studies have not.
The other limitation of this study remained our small sample size. The limited number of B1, B2, and B3 glenoids prohibited statistical analysis of these glenoid morphologies. However, we wanted to study both CT and MRIs in the same patients to evaluate both static and dynamic stabilizers of the shoulder joint, which has not been done previously. The inclusion of patients with only CT scans would have increased sample size, and using published protocols, information on the rotator cuff muscles could have been obtained, but we thought it prudent to include tendinous integrity to examine all possible anatomic effectors of shoulder stability and wear simultaneously, despite reduced sample size, to avoid misinterpreting any potential relationships $[25,26]$. We were also limited by the cross-sectional nature of the study. A prospective study analyzing the natural course of glenoid wear in the setting rotator cuff pathology would be necessary to fully assess the temporal sequence of pathologic change and causality.

\section{Conclusion}

In conclusion, the proposed measurement protocol is a reliable and valid method of quantifying glenoid bone loss. Increasing glenoid anteversion and anterior glenoid bone loss correlates to increased anterior humeral subluxation. Conversely, posterior bone loss correlates to increased posterior humeral subluxation. Humeral head subluxation is not significantly affected by rotator tendinopathy or muscle atrophy. However, fatty infiltration of the subscapularis muscle is correlated with increased anterior and central glenoid bone loss. Further studies are required to determine the temporal sequence of events in glenoid bone loss and subscapularis fatty infiltration and whether or not subscapularis atrophy and/or strengthening could serve as independent prognostic factors affecting TSA outcomes.

Compliance with ethical standards This was a crosssectional study performed in compliance with HIPAA regulations and was approved by our tertiary care institutional review board. Informed consent was waived due to the retrospective nature of the study.

\section{References}

1. Kerr R, Resnick D, Pineda C, Haghighi P. Osteoarthritis of the glenohumeral joint: a radiologic-pathologic study. AJR Am J Roentgenol. 1985;144(5):967-72.

2. Petersson CJ. Degeneration of the gleno-humeral joint: an anatomical study. J Acta Orthopaedica Scandinavica. 1983;54(2):277-83.

3. Murphy LB, Cisternas MG, Pasta DJ, Helmick CG, Yelin EH. Medical expenditures and earnings losses among US adults with arthritis in 2013. Arthritis Care Res. 2018;70(6):869-76.

4. Dillon MT, Chan PH, Inacio MC, Singh A, Yian EH, Navarro RA. Yearly trends in elective shoulder arthroplasty, 2005-2013. Arthritis Care Res. 2017;69(10):1574-81. 
5. Westermann RW, Pugely AJ, Martin CT, Gao Y, Wolf BR, Hettrich CM. Reverse shoulder arthroplasty in the United States: a comparison of national volume, patient demographics, complications, and surgical indications. Iowa Orthopedic J. 2015;35:1-7.

6. Walch G, Moraga C, Young A, Castellanos-Rosas J. Results of anatomic nonconstrained prosthesis in primary osteoarthritis with biconcave glenoid. J Shoulder Elb Surg. 2012;21(11):1526-33.

7. Young AA, Walch G, Pape G, Gohlke F, Favard L. Secondary rotator cuff dysfunction following total shoulder arthroplasty for primary glenohumeral osteoarthritis: results of a multicenter study with more than five years of follow-up. J Bone Joint Surg Am. 2012;94(8):685-93.

8. Donohue KW, Ricchetti ET, Ho JC, Iannotti JP. The association between rotator cuff muscle fatty infiltration and glenoid morphology in glenohumeral osteoarthritis. J Bone Joint Surg Am. 2018;100(5):381-7.

9. Bercik MJ, Kruse K 2nd, Yalizis M, Gauci MO, Chaoui J, Walch G. A modification to the Walch classification of the glenoid in primary glenohumeral osteoarthritis using three-dimensional imaging. J Shoulder Elb Surg. 2016;25(10):1601-6.

10. Rouleau DM, Kidder JF, Pons-Villanueva J, Dynamidis S, Defranco M, Walch G. Glenoid version: how to measure it? Validity of different methods in two-dimensional computed tomography scans. J Shoulder Elb Surg. 2010;19(8):1230-7.

11. Walch G, Badet R, Boulahia A, Khoury A. Morphologic study of the glenoid in primary glenohumeral osteoarthritis. J Arthroplast. 1999; 14(6):756-60.

12. Nowak DD, Gardner TR, Bigliani LU, Levine WN, Ahmad CS. Interobserver and intraobserver reliability of the Walch classification in primary glenohumeral arthritis. J Shoulder Elb Surg. 2010;19(2):180-3.

13. Scalise JJ, Codsi MJ, Brems JJ, Iannotti JP. Inter-rater reliability of an arthritic glenoid morphology classification system. J Shoulder Elb Surg. 2008;17(4):575-7.

14. Goutallier D, Postel J-M, Bernageau J, Lavau L, Voisin M-C. Fatty muscle degeneration in cuff ruptures. Pre-and postoperative evaluation by CT scan. J Clinical orthopaedics. 1994;304:78-83.

15. Warner JJ, Higgins L, Parsons IM, Dowdy P. Diagnosis and treatment of anterosuperior rotator cuff tears. J Shoulder Elb Surg. 2001;10(1):37-46.

16. Neyton L, Gauci MO, Deransart P, Collotte P, Walch G, Athwal GS. Three-dimensional characterization of the anteverted glenoid (type
D) in primary glenohumeral osteoarthritis. J Shoulder Elb Surg. 2019;28(6):1175-82.

17. van de Bunt F, Pearl ML, Lee EK, Peng L, Didomenico P. Glenoid version by CT scan: an analysis of clinical measurement error and introduction of a protocol to reduce variability. Skelet Radiol. 2015;44(11):1627-35.

18. Parada SA, Eichinger JK, Dumont GD, Burton LE, Coats-Thomas MS, Daniels SD, et al. Comparison of glenoid version and posterior humeral subluxation in patients with and without posterior shoulder instability. Arthroscopy. 2017;33(2):254-60.

19. Hoenecke HR Jr, Tibor LM, D'Lima DD. Glenoid morphology rather than version predicts humeral subluxation: a different perspective on the glenoid in total shoulder arthroplasty. J Shoulder Elb Surg. 2012;21(9):1136-41.

20. Aygun U, Calik Y, Isik C, Sahin H, Sahin R, Aygun DO. The importance of glenoid version in patients with anterior dislocation of the shoulder. J Shoulder Elb Surg. 2016;25(12):1930-6.

21. Di Giacomo G, Piscitelli L, Pugliese M. The role of bone in glenohumeral stability. EFORT Open Rev. 2018;3(12):632-40.

22. Matsen FA 3rd, Chebli C, Lippitt S. Principles for the evaluation and management of shoulder instability. J Bone Joint Surg Am. 2006;88(3):648-59.

23. Werthel JD, Schoch B, Frankle M, Cofield R, Elhassan BT. Shoulder arthroplasty for sequelae of obstetrical brachial plexus injury. J Hand Surg. 2018;43(9):871.e871-7.

24. van Gelein Vitringa VM, van Kooten EO, Mullender MG, van Doorn-Loogman MH, van der Sluijs JA. An MRI study on the relations between muscle atrophy, shoulder function and glenohumeral deformity in shoulders of children with obstetric brachial plexus injury. J Brachial Plex Peripher Nerve Inj. 2009;4:9.

25. van de Sande MA, Stoel BC, Obermann WR, Tjong a Lieng JG, Rozing PM. Quantitative assessment of fatty degeneration in rotator cuff muscles determined with computed tomography. Investig Radiol 2005; 40(5):313-319.

26. Terrier A, Ston J, Dewarrat A, Becce F, Farron A. A semiautomated quantitative $\mathrm{CT}$ method for measuring rotator cuff muscle degeneration in shoulders with primary osteoarthritis. Orthop Traumatol Surg Res. 2017;103(2):151-7.

Publisher's note Springer Nature remains neutral with regard to jurisdictional claims in published maps and institutional affiliations. 